\title{
Experimental Study on Thermal and Catalytic Decomposition of a Dual-Mode Ionic Liquid Propellant
}

\author{
He Gao ${ }^{1,2}$, Shaolong $\mathrm{Li}^{3}$, Zhaopu $\mathrm{Yao}^{1,2^{*}}$ and Shuiqing $\mathrm{Li}^{3}$ \\ ${ }^{1}$ Beijing Institute of Control Engineering, Beijing 100190, China; \\ ${ }^{2}$ Beijing Engineering Research Center of Effificient and Green Aerospace Propulsion Technology, Beijing, 100190, China; \\ ${ }^{3}$ Department of Energy and Power Engineering, Tsinghua University, Beijing, 100084, China
}

\begin{abstract}
With the increasing of space activities and people's awareness of environmental protection, it is necessary to develop a new non-toxic space propulsion system with high performance. Hydroxylammonium nitrate (HAN)/1-ethyl-3-methyl-imidazolium ethylsulfate([Emim][EtSO4]) blend ionic liquid propellant is a potential replacement with non-toxic and high-performance characteristics for hydrazine type liquid propellants, which can be used in both chemical and electrical propulsion system. This paper introduced the thermogravimetric experimental analysis (TGA-DSC) results of HAN/[Emim][EtSO4] ionic liquid propellant with the thermal decomposition and catalytic decomposition process. Its mass-loss process and exothermic process under different reaction conditions at a heating rate of $5 \mathrm{~K} / \mathrm{min} \sim 15 \mathrm{~K} / \mathrm{min}$ were studied. Generally, the mass-loss results showed that there were four characteristic stages during the decomposition process of the HAN/[Emim][EtSO4] ionic liquids, which were the evaporation of the water solvent, decomposition of the HAN component, further decomposition of the [Emim][EtSO4], and slow loss of the residual substances. At the same time, two exothermic peaks were observed, which respectively corresponded to the decomposition of HAN and the further decomposition of [Emim][EtSO4]. Using catalyst can significantly reduce the decomposition temperature of the propellant and the residual mass. The contents in this paper proved that this propellant had a good application prospect within the catalytic ignition aerospace thruster.
\end{abstract}

\section{Introduction}

The hydrazine and hydrazine-based liquid propellants play dominant roles for aerospace propulsion in the past few decades. However, due to its toxic characterizes, it also brings significant health hazards. With the increasing of space activities and people's awareness of environmental protection, it is necessary to develop a new non-toxic space propulsion system with high performance. Ionic liquid has the advantages of low vapor pressure, environmentally friendly and low toxicity. The multi-component ionic liquid is a potential replacement for hydrazine type liquid propellants by introducing energetic components to improve the performance[1]. Ionic liquid can also be used in electric propulsion system due to its high degree of ionization and easy interaction with electric field, so it is possible to apply the same ionic liquid to both the chemical and electric propulsion systems and realize the dual-mode aerospace propulsion technology.

The research of dual - mode ionic liquid propulsion technology is on the rise in the world. Hydroxylammonium nitrate (HAN)/1-ethyl-3-methylimidazolium ethyl-sulfate([Emim][EtSO4]) blend ionic liquid propellant is a promising propellant for dual-mode propulsion systems with non-toxic, high density and high degree of ionization characteristics. In this propellant, HAN is the oxidizer and can be miscible with the fuel [Emim][EtSO4] by adding a little water to form premixed multi-component ionic liquid propellant. The propellant can be used in chemical propulsion based on catalytic ignition or in electric propulsion with electrospray operation mode. Some relevant research has been carried out on the characteristics and performance of this propellant. Rovey's team from Missouri University of Science and Technology had conducted exploratory research on the application prospect of this ionic liquid in the dual-mode propulsion system, demonstrating the superiority and feasibility of this dualmode ionic liquid propulsion system[2-4]. Berg and Donius compared the performance of different kinds of ionic liquid propellants. And the results showed that $\mathrm{HAN} /[\mathrm{Emim}][\mathrm{EtSO} 4]$ formula was a promising dualmode ionic liquid propellant[5-7]. Also, Berg studied the thermal decomposition process of HAN/[Emim][EtSO4] ionic liquid propellant on different metal surfaces by measuring the pressure changes in the micro-reactor, and proved that platinum had a better catalytic effect on $\mathrm{HAN} /[\mathrm{Emim}][\mathrm{EtSO} 4]$ ionic liquid propellant[8,9]. Moreover, they found that the thermal stability of $\mathrm{HAN} /[\mathrm{Emim}][\mathrm{EtSO} 4]$ ionic liquid propellant could be reduced by adding ferrofluids[10]. Berg also studied the ignition performance of $\mathrm{HAN} /[\mathrm{Emim}][\mathrm{EtSO} 4]$ ionic liquid propellant in capillary tubes, and proved that this ionic liquid could realize stable ignition in platinum capillary tubes[11]. Berg and Wainwright measured the emission characteristics of HAN/[Emim][EtSO4] ionic liquid propellant in electric field by angle-resolved 
method and mass Spectroscopy[12,13], which showed the potential of this propellant in dual-mode space propulsion technology. Mundahl and Rasmont measured the linear burning rate of HAN/[Emim][EtSO4] ionic liquid propellant, and found that the linear burning rate was strongly related to environmental pressure. At lower pressure range $(0.5 \sim 3 \mathrm{MPa})$, the linear burning rate increased almost linearly with the increase of pressure.And at higher pressure range $(3 \sim 10 \mathrm{MPa})$, the increase rate slowed down significantly[14,15]. In China, Dalian Institute of Chemical Physics studied the formulation of $\mathrm{HAN} /[\mathrm{Emim}][\mathrm{EtSO} 4]$ ionic liquid propellant. And an ideal formula of this propellant was obtained for further research[16].

As a novel ionic liquid propellant, there is few researches on the characteristics of thermal decomposition and catalytic decomposition process of $\mathrm{HAN} /[\mathrm{Emim}][\mathrm{EtSO} 4]$ ionic liquid propellant. In this paper, by the thermogravimetric analysis method, the mass loss and heat release during the thermal decomposition and catalytic decomposition process of $\mathrm{HAN} /[$ Emim] $]$ EtSO4] ionic liquid propellant was studied with detail. The effects of heating rate, mass ratio of propellant to catalyst on the reaction process were studied. The contents in this paper proved that this propellant had a good application prospect within the catalytic ignition aerospace thruster.

\section{Experimental method}

\subsection{Propellants and catalysts}

The propellant and catalyst used in this study are described in reference [16]. The propellant is composed of $50 \%$ wt HAN, $40 \%$ wt [Emim][EtSO4], 10\%wt H2O and a few chemical additives. The catalyst is Ir - based catalyst supported by $\mathrm{A} 12 \mathrm{O} 3$. The catalyst mainly acts on the HAN of the propellant. So, the chemical reaction process of the propellant can be generally described as two steps. First, with the effects of heating or catalyst, the HAN in the propellant decomposes and releases heat. And then, the oxidation substances generated by the HAN decomposition react with the [Emim][EtSO4] to promote the further decomposition of the [Emim][EtSO4] in the propellant and releases heat again.

\subsection{Experiment setup}

A STA449F3 thermogravimetric analyzer-differential scanning calorimeter(TGA-DSC, Netzsch Co.) was used in this study. The temperature resolution of this analyzer can reach $0.001 \mathrm{~K}$ and the mass resolution can reach $0.1 \mu \mathrm{g}$. The experiment schematic is shown in Fig. 1. The results obtained from the TGA-DSC experiments enable us to investigate the mass loss and the heat release during the propellant decomposition process, which help us to evaluate the reaction characteristics of the propellant and effects of the catalyst.

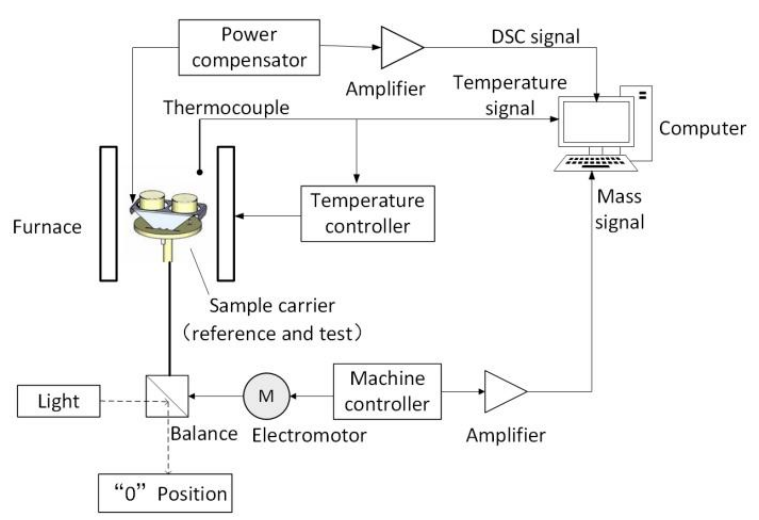

Fig.1. TGA-DSC system schematic diagram

For TGA-DSC experiments, it's very necessary to control the sample quality and heating rate during the experimental process. Keeping the sample mass in a small range can prevent splashing and make the sample more easily reach the thermal equilibrium state, which reduces the systematic error of the experiments. Too high heating rate will increase the risk of propellant deflagration. And low heating rate will slow down some physical and chemical process and blur the mass loss steps. During the experiments in this paper, Alumina crucibles were used for the experiments. And the heating temperature range was $30 \sim 500^{\circ} \mathrm{C}$ with a heating rate of $5 \mathrm{~K} / \mathrm{min}, 10 \mathrm{~K} / \mathrm{min}$ and $15 \mathrm{~K} / \mathrm{min}$ under a nitrogen flow (80 $\mathrm{mL} / \mathrm{min})$. The propellant quality was controlled as $4.5 \mathrm{mg}$ in each experiment. And the mass fraction of propellant $\omega$ in the sample was defined as:

$$
\omega=\frac{M p}{M p+M c}
$$

$M p$ was the propellant sample mass and $M c$ was the catalyst mass. The parameters controlled in this study included the heating rate and the mass fraction of the propellant $\omega$.

The experiment parameters in each experiment case are shown in table 1. Case 1 3 studied the thermal decomposition process of the propellant without catalyst under different heating rates. Fixed the propellant quality as $4.5 \mathrm{mg}$ and the $\omega$ value as about $55 \%$, Case $4 \sim 6$ studied the catalytic decomposition process of propellant under different heating rates. Case 7 10 studied the catalytic decomposition process of propellant with various propellant mass fraction $\omega$ under a fixed propellant quality as $4.5 \mathrm{mg}$ and a fixed heating rate of $15 \mathrm{~K} / \mathrm{min}$.

Table 1. experiment parameters in each case

\begin{tabular}{llll}
\hline Case & Heating rate $/(\mathrm{K} / \mathrm{min})$ & Catalyst & $\omega$ \\
\hline 1 & 5 & NO & 100.00 \\
2 & 10 & NO & 100.00 \\
3 & 15 & NO & 100.00 \\
4 & 5 & YES & 54.68 \\
5 & 10 & YES & 55.08 \\
6 & 15 & YES & 54.57 \\
7 & 15 & YES & 31.96 \\
8 & 15 & YES & 45.97 \\
9 & 15 & YES & 62.63 \\
10 & 15 & YES & 89.98 \\
\hline
\end{tabular}




\section{Results and discussions}

\subsection{The general reaction characteristics of the propellant}

First, the general reaction characteristics of the propellant were observed in four test tubes at different reaction conditions. The propellant was relatively stable at room temperature without catalyst. And no bubbles or other reaction phenomena were observed, as shown in fig. 1(a). If the catalyst was added, it was observed that a large number of small bubbles were generated in the propellant at room temperature. However, the color of the liquid didn't change significantly, as shown in fig. 1(b). And in fig. 1(c), the propellant was heated to $250^{\circ} \mathrm{C}$ without catalyst. A large number of bubbles were generated quickly and the color of the solution turned to yellow. In fig. 1(d), combined the effects of catalyst and heating, a large amount of gas was generated violently and even spattered. But no ignition was observed.

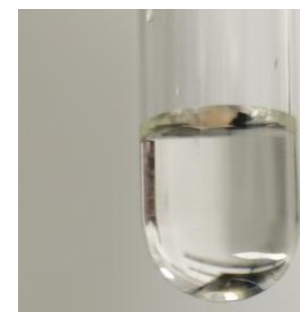

(a)

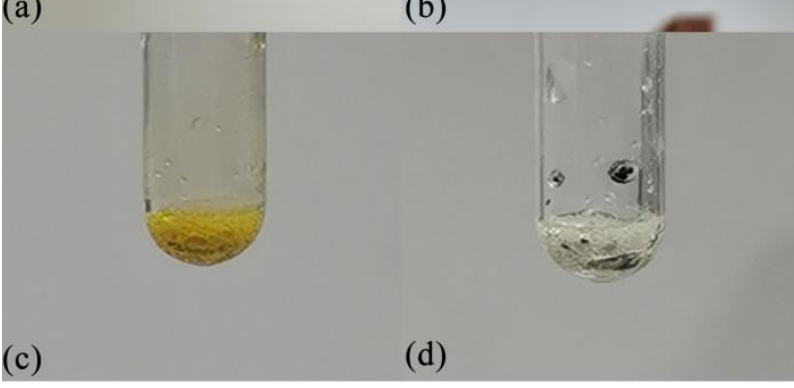

Fig.2. The decomposition process of propellant under different conditions,

(a) Room Temperature, NO Catalyst, (b) Room Temperature, Catalyst, (c) $250^{\circ} \mathrm{C}, \mathrm{NO}$ Catalyst, (d) $250^{\circ} \mathrm{C}$, Catalyst

\subsection{The thermal decomposition process of the propellant}

Case 1 3 studied the thermal decomposition process of the propellant without catalyst under different heating rates. The TG and DSC results were shown in fig. 3 and fig. 4, respectively. According to the results in fig. 3, generally, the thermal decomposition process of HAN/[Emim][EtSO4] ionic liquid propellant was a continuous mass loss process, which included four characteristic stages. Taking the TG curve of the $5 \mathrm{~K} / \mathrm{min}$ case in fig. 3 as an example, in the stage1, the mass loss rate was very low originally and increased slowly until the mass loss reached about $20 \% \sim 30 \%$. The mass loss was mainly caused by the evaporation of water in propellant. But it should be noted that the value of the mass loss was greater than the proportion of water in the propellant, which could be explained by the corresponding DSC curves as shown in fig. 4. During the heating process with heating rate of $5 \mathrm{~K} / \mathrm{min}$, the exothermic effect became obvious at about $200^{\circ} \mathrm{C}$, which was caused by the thermal decomposition of the HAN in the propellant and the release of heat. Due to the thermal decomposition of the HAN, the additional mass loss was also caused. The mass loss rate of the stage 2 was obviously higher than the stage 1 . In this stage, the decomposition of HAN mainly occurred as well as the preliminary decomposition of [Emim][EtSO4]. The total mass loss of the first and second stages was about $50 \sim 60 \%$. Followed by, the stage 3 was a rapid mass losing stage in which the mass loss of the sample reached more than 30\% within few minutes. Further decomposition of [Emim][EtSO4] occurred in this stage. The remaining mass was about $10 \%$. And the decomposition entered the stage 4 , in which the residual material decomposed slowly. And the final remaining mass was about $5-8 \%$.

With other heating rates as $10 \mathrm{~K} / \mathrm{min}$ and $15 \mathrm{~K} / \mathrm{min}$, the decomposition process had the similar trend. However, the mass loss at different stages varied due to different heating rate. It could be conclude that with the increase of heating rate, the mass loss in the stage 2 increased, while the mass loss in the stage 1 and stage 3 decreased. That was because the increase of the heating rate accelerated the evaporation of water and promoted the transition of reaction to the stage 2 . The decomposition of HAN produced a large number of oxidizing substances which also promoted the preliminary decomposition of [Emim][EtSO4]. This effect increased the mass loss in the stage 2 and reduced the mass loss in the stage 1 and stage 3 . Based on the experimental results, the heating rate didn't affect the residual mass obviously.

It could be observed in the DSC curves in fig. 4 that there were two exothermic peaks in each curve within the temperature range of $180 \sim 300^{\circ} \mathrm{C}$, which corresponded to the decomposition process of HAN and [Emim][EtSO4], respectively. DSC results showed that with the increase of heating rate, the peak value and area of the exothermic peak1 gradually increased. As discussed above, that was because more HAN was decomposed in stage 2 and more heat was released due to the accelerating of HAN decomposition. 


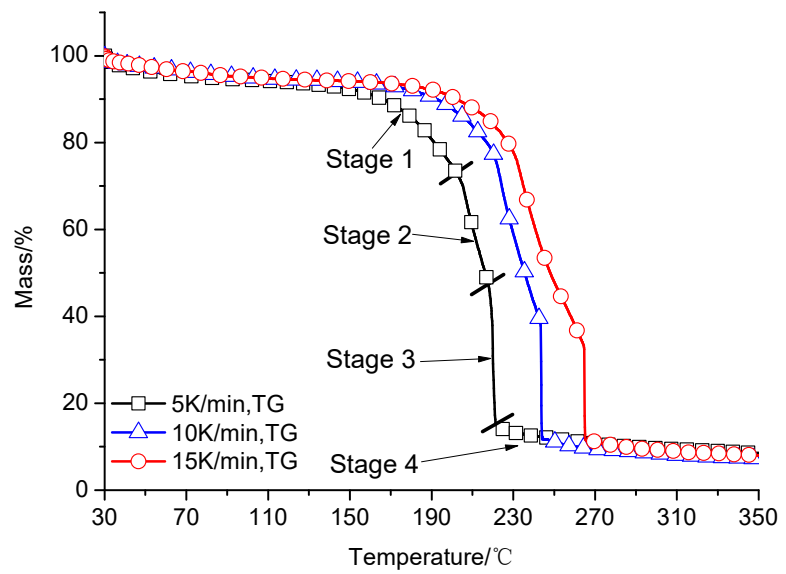

Fig.3. TG curves of thermal decomposition with different heating rates

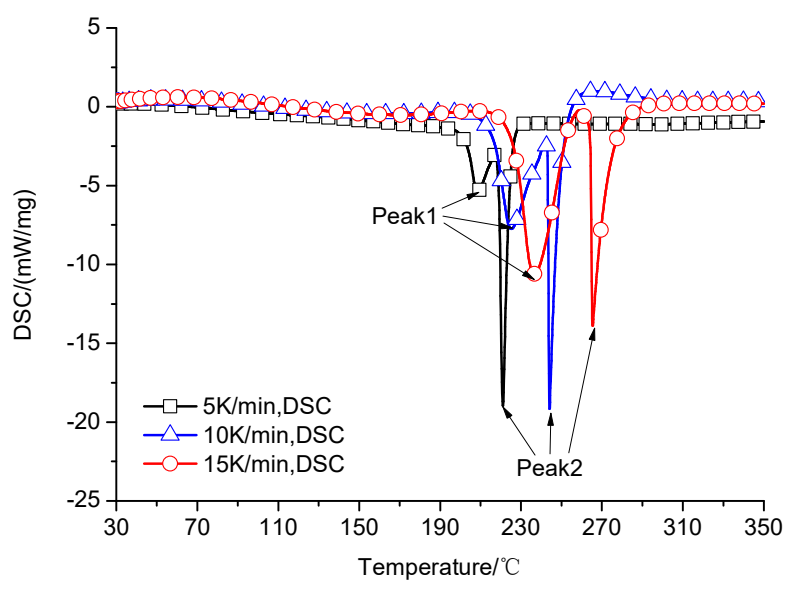

Fig.4. DSC curves of thermal decomposition with different heating rates

\subsection{The catalytic decomposition process of propellant}

Fixed the propellant quality as $4.5 \mathrm{mg}$ and the $\omega$ value as about $55 \%$, Case $4 \sim 6$ studied the catalytic decomposition process of propellant under different heating rates. Fig. 5 and 6 showed the TG and DSC results of the catalytic decomposition compared with the thermal decomposition, respectively.

As shown in fig. 5, the catalytic mass-loss process of propellants with different heating rates revealed a common feature. Compared with the thermal decomposition process of the propellant, the adding of catalyst changed the characteristics of the four stages in the mass loss process. The stages of water evaporation and HAN decomposition almost merged. That was because the catalyst reduced the activation energy of HAN decomposition and also reduced the decomposition temperature of HAN. The catalytic decomposition process of HAN partially coincided with the evaporation process of water. Moreover, in the catalytic decomposition experiments, the residual mass was about $3 \%$ lower than that of the thermal decomposition experiments under the corresponding heating rates.
Considering the different heating rates and the corresponding temperature of stage transition, it could be concluded that a higher heating rate accelerated the whole catalytic decomposition process of propellant.

The catalyst also affected the exothermal peaks of the DSC curves in fig. 6. Compared with the thermal decomposition process of the propellant, after the use of catalyst, the first exothermic peak of the DSC curve was significantly expanded due to the reduction of the initial decomposition temperature of HAN. And the corresponding temperatures of the two exothermic peak values were significantly decreased with the same heating rate. At the heating rate of $5 \mathrm{~K} / \mathrm{min}$, the first exothermic peak of propellant catalytic decomposition reaction was almost even because of the slower heating rate and longer heating time. When the heating rate increased to $15 \mathrm{~K} / \mathrm{min}$ as labeled in fig. 6 , the exothermic peaks with catalyst became more obvious and could be compared with the ones of thermal decomposition with details. The two exothermic peaks with catalyst moved to lower temperature range compared to the exothermic peaks under thermal decomposition. With the adding of catalyst, the peak value of the first exothermic peak was smaller than the second one. However, the trend was contrary for the two exothermic peaks under thermal decomposition as labeled in fig. 6 .

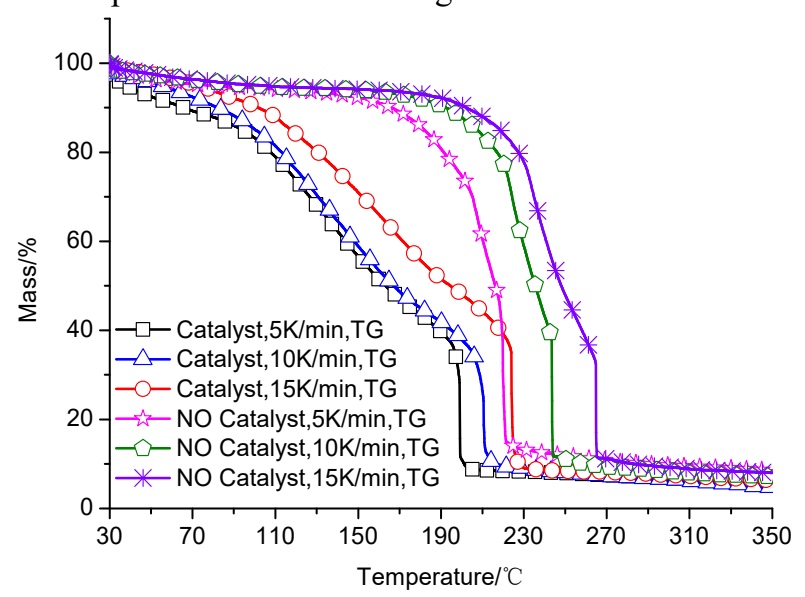

Fig.5. TG curves of catalytic decomposition compared with thermal decomposition

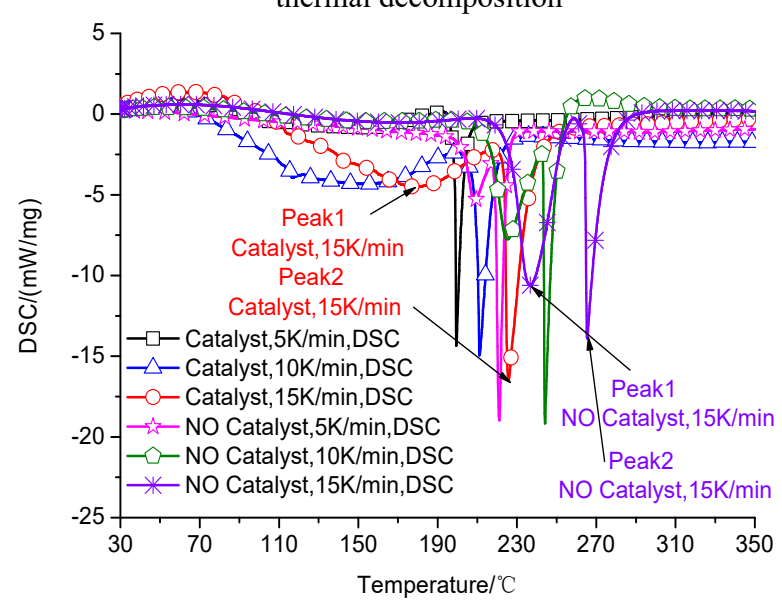

Fig.6. DSC curves of catalytic decomposition compared with thermal decomposition 


\subsection{Effects of the mass fraction of propellant $\omega$}

In order to study the effects of the mass ratio of propellant to catalyst on the catalytic decomposition characteristics of the propellant, the mass fraction of propellant $\omega$ varied by fixing the mass of the propellant to $4.5 \mathrm{mg}$ and changing the mass of the catalyst. Case 7 10 in table 1 were carried out. Fig. 7 and fig. 8 showed the TG curves and DSC curves of the catalytic decomposition process in case 7 10, respectively.

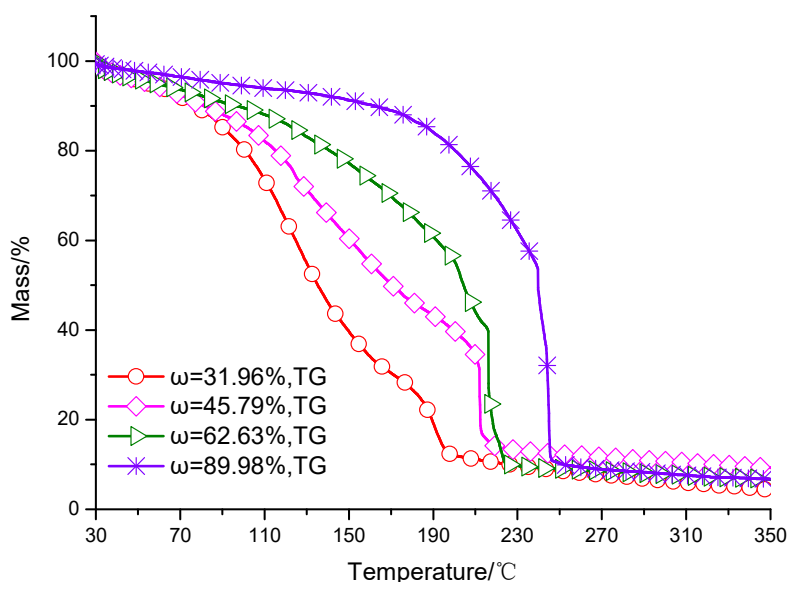

Fig.7. TG curves of catalytic decomposition with different $\omega$, $15 \mathrm{~K} / \mathrm{min}$

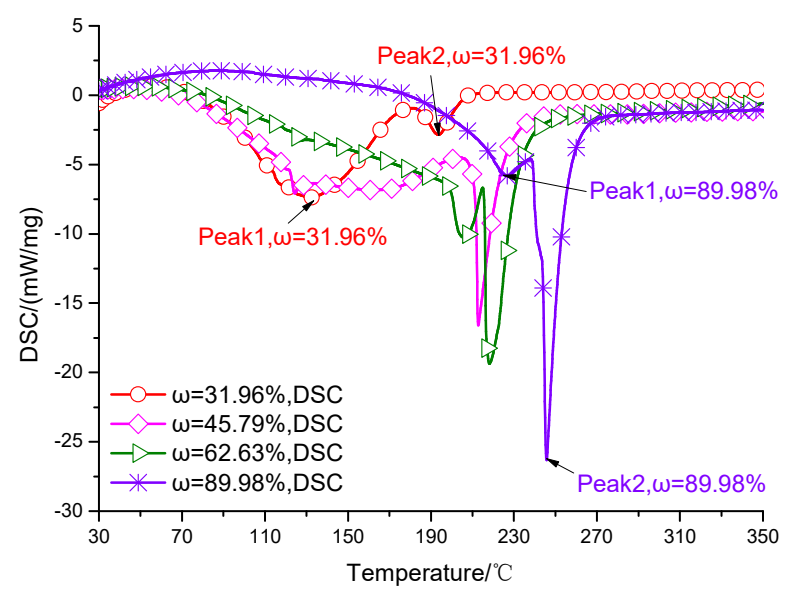

Fig.8. DSC curves of catalytic decomposition with different $\omega, 15 \mathrm{~K} / \mathrm{min}$

It could be observed from fig. 7 that, under the reaction condition with less catalyst $(\omega=89.98 \%)$, the overall characteristics of the mass loss curve was similar to the one with the thermal decomposition process. The four characteristic stages of mass-loss were relatively obvious. With the increase of catalyst, the characteristic stages of the propellant mass loss process merged gradually. When $\omega=31.96 \%$, the slope of the first three characteristic processes of the mass loss curve was very similar. And it was the fastest to enter the fourth stage of slow residual mass loss. That indicated that with the same heating rate, increasing the amount of the catalyst was more conducive to the propellant catalytic decomposition reaction. With the decrease of $\omega$, the mass ratio of catalyst keeps increasing. DSC results in fig. 8 showed that, the corresponding temperature of the exothermic peaks in each DSC curve kept moving to a lower temperature direction. It indicated that the increase of catalyst mass ratio promoted the whole catalytic decomposition process of propellant. At the same time, as the value of $\omega$ decreased from $89.98 \%$ to $31.96 \%$, the shape of the two exothermic peaks in the DSC curves also changed. With the decrease of $\omega$ value, the first exothermic peak of the catalytic reaction process gradually extended, which corresponded to the gradual merge of the characteristic stage of the propellant mass loss process in fig. 7. When $\omega=89.98 \%$, the peak value of the second exothermic peak of DSC curve was larger than that of the first exothermic peak. However, when $\omega=31.96 \%$, the peak value of the second exothermic peak of DSC curve was smaller than that of the first exothermic peak. This also indicated that the increase of catalyst made the overall process of catalytic decomposition accelerate.

Observing the crucibles after the experiments as shown in fig. 9, it could be found that when the mass ratio of catalyst was low $(\omega=89.98 \%)$, the catalyst couldn't decompose all the propellant and the incompletely reacted propellant formed coke on the surface of the catalyst and sintered the catalyst grains, as shown in fig. 9(a). When the mass ratio of catalyst was high $(\omega=31.96 \%)$, the catalyst could decompose all the propellant and the catalyst particles were separate, as shown in fig. 9(b).

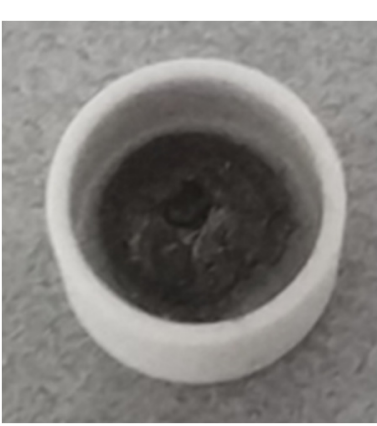

(a)

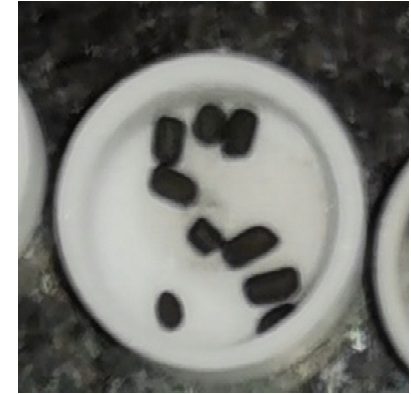

(b)
Fig.9. The catalyst particles after experiment, (a) $\omega=89.98 \%$, (b) $\omega=31.96 \%$

\section{Conclusions}

In this paper, the thermal and catalytic decomposition processes of the HAN/[Emim][EtSO4] ionic liquid propellant was investigated experimentally with detail by the TGA-DSC method. It could be concluded that the thermal decomposition process of the propellant could be divided into four characteristics stages, of which the second and third stages were the decomposition processes of HAN and [Emim][EtSO4]. They were also the main mass-loss and exothermic stages during the whole decomposition process. The adding of catalyst could merge the decomposing stages of the propellant compared to the thermal decomposition and reduce the 
residual substances. And the use of the catalyst could effectively reduce the decomposition temperature of propellant and accelerate the overall process of catalytic decomposition. The contents presented in this paper can be expected to have practical applications in the development of the HAN/[Emim][EtSO4] ionic liquid propulsion techniques.

\section{References}

1. A E Visser, N J Bridges and R D Rogers Ionic Liquids: Science and Applications (USA:American Chemical Society,2012) chapter 5 pp 173-214

2. J Rovey, C T Lyne, A J Mundahl and N Rasmont AIAA Propulsion and Energy 2019 Forum (2019)

3. S P Berg Missouri University Of Science And Technology (2017)

4. S P Berg and J L Rovey J. Spacecr. Rockets 54(3) 592-601 (2017)

5. B R Donius and J L Rovey J. Spacecr. Rockets 48(1) 110-23(2011)

6. $\mathrm{S}$ P Berg and J L Rovey 49th AIAA/ASME/SAE/ASEE Joint Propulsion Conference (2013)
7. A J Mundahl, S P Berg, J L Rovey, M Huang and K Woelk 53rd AIAA/SAE/ASEE Joint Propulsion Conference (2017)

8. S P Berg and J L Rovey 52nd AIAA/SAE/ASEE Joint Propulsion Conference (2016)

9. S P Berg and J L Rovey J. Propul. Powe 29(1) 12535 (2013)

10. S P Berg, B Coleman and J L Rovey 50th AIAA/SAE/ASEE Joint Propulsion Conference (2014)

11. S P Berg and J L Rovey 52nd AIAA/SAE/ASEE Joint Propulsion Conference (2017)

12. S P Berg, J L Rovey,B D Prince,S W Miller and R Bemish 51st AIAA/SAE/ASEE Joint Propulsion Conference (2015)

13. M J Wainwright, J L Rovey,S W Miller, B D Prince and S P Berg 2018 Joint Propulsion Conference (2018)

14. N Rasmont, E J Broemmelsiek and J L Rovey Combust. Flame 219 212-24 (2020)

15. A J Mundahl, J L Rovey and S P Berg 2018 Joint Propulsion Conference (2018)

16. W T Wang, Z Y Ma, W M Cong, W S Zhang, L G Xia, X D Wang J. Propul. Technol. 41(20) 45560(2020) Chinese 